\title{
Differential efficiency among DNA extraction methods influences detection of the amphibian pathogen Batrachochytrium dendrobatidis
}

\author{
M. C. Bletz ${ }^{1,2, *}$, E. A. Rebollar ${ }^{1}$, R. N. Harris ${ }^{1}$ \\ ${ }^{1}$ Department of Biology, James Madison University, 951 Carrier Drive, Harrisonburg, VA 22807, USA \\ ${ }^{2}$ Present address: TU Braunschweig, Zoological Institute, Mendelssohnstr. 4, 38106 Braunschweig, Germany
}

\begin{abstract}
Chytridiomycosis, caused by the fungal pathogen Batrachochytrium dendrobatidis $(B d)$, is responsible for massive declines and extinctions of amphibians worldwide. The most common method for detecting $B d$ is quantitative polymerase chain reaction (qPCR). qPCR is a highly sensitive detection technique, but its ability to determine the presence and accurately quantify the amount of $B d$ is also contingent on the efficiency of the DNA extraction method used prior to PCR. Using qPCR, we compared the extraction efficiency of 3 different extraction methods commonly used for $B d$ detection across a range of zoospore quantities: PrepMan Ultra Reagent, Qiagen DNeasy Blood and Tissue Kit, and Mobio PowerSoil DNA Isolation Kit. We show that not all extraction methods led to successful detection of $B d$ for the low zoospore quantities and that there was variation in the estimated zoospore equivalents among the methods, which demonstrates that these methods have different extraction efficiencies. These results highlight the importance of considering the extraction method when comparing across studies. The Qiagen DNeasy kit had the highest efficiency. We also show that replicated estimates of less than 1 zoospore can result from known zoospore concentrations; therefore, such results should be considered when obtained from field data. Additionally, we discuss the implications of our findings for interpreting previous studies and for conducting future $B d$ surveys. It is imperative to use the most efficient DNA extraction method in tandem with the highly sensitive qPCR technique in order to accurately diagnose the presence of $B d$ as well as other pathogens.
\end{abstract}

KEY WORDS: qPCR · Qiagen · DNeasy kit $\cdot$ PrepMan Ultra $\cdot$ Chytridiomycosis

Resale or republication not permitted without written consent of the publisher

\section{INTRODUCTION}

Amphibians worldwide are threatened by the disease chytridiomycosis, which is caused by the fungal pathogen Batrachochytrium dendrobatidis $(B d)$ (Hoffmann et al. 2010, Fisher et al. 2012). This pathogen is linked to the declines and extinctions of numerous amphibian species (Wake \& Vredenburg 2008, Fisher et al. 2009, Crawford et al. 2010). It is critical to be able to detect the presence of $B d$ with high sensitivity to accurately determine the patho- gen's distribution and to track disease dynamics. This will be especially important when infection intensities are expected to be low, which occurs (1) where environmental conditions for $B d$ are not optimal such as lowland tropical areas (Rebollar et al. 2014), (2) in resistant species, such as some species in North America (e.g. Rothermel et al. 2008), and (3) at the early onset of $B d$ 's arrival in naïve areas. Early detection of emergence in naïve areas is crucial in order to quickly implement disease mitigation tactics. 
The methods for detecting $B d$ have progressed from histology to traditional PCR (Annis et al. 2004) to quantitative PCR (qPCR) (Boyle et al. 2004) within the last $15 \mathrm{yr}$. The ability to determine the presence of $B d$ and to estimate its abundance on individuals depends on DNA extraction efficiency (i.e. the ability to extract the DNA present in the sample) and PCR sensitivity (i.e. the ability to amplify the DNA templates present in the extract). Detection is also contingent on the ability of the extraction method to purify samples and remove enzyme inhibitors. In recent years, qPCR has been the method of choice and has been shown to be a highly sensitive technique (Boyle et al. 2004); however, the efficiency of the DNA extraction method has not been evaluated for the common extraction methods used for $B d$ detection. It is essential to choose the extraction method with the highest efficiency in order to accurately determine the presence and intensity of the chytrid fungus. Low extraction efficiency could result in false negatives, which could have important consequences for amphibian conservation and disease mitigation decisions when $B d$ arrives in an area with susceptible amphibian species.

Several different methods, including PrepMan Ultra Reagent, Qiagen DNeasy Blood and Tissue Kit, and Mobio PowerSoil DNA Isolation Kit, are commonly used for $B d$ detection. PrepMan Ultra Reagent with the addition of zirconium beads, which was suggested by Boyle et al. (2004) for amphibian swab detection of $B d$, has been a common method of choice due to its cost effectiveness and ease of use (Kriger \& Hero 2007, Duncan Pullen et al. 2010, Searle et al. 2011, Swei et al. 2011, Vredenburg et al. 2012). The Qiagen DNeasy Blood and Tissue Kit has also been used because it minimizes the potential for PCR inhibition (Harris et al. 2009, Cheng et al. 2011, Muletz et al. 2012, Rasmussen et al. 2012, Chatfield et al. 2013, Rebollar et al. 2014). Recently this method was found to have the best balance of inhibition removal and cost effectiveness (Kosch \& Summers 2013). Researchers also interested in microbial communities on amphibian skin or environmental samples have chosen to use Mobio kits including the Ultra Clean Microbial DNA Isolation Kit and PowerSoil DNA Isolation Kit because they are very effective at removing PCR inhibitors (Kirshtein et al. 2007, McKenzie et al. 2012).

Here we compared the efficiency of 3 DNA extraction methods by evaluating the detection (presence/ absence) and intensity (estimated zoospore equivalents) of $B d$, through the use of qPCR. In addition, we discuss the implications of our findings for diagnos- ing the presence of the pathogen and make recommendations on the best detection techniques.

\section{MATERIALS AND METHODS}

\section{Harvesting and quantifying zoospores}

$B d$ strain JEL 423, an isolate collected from an individual lemur frog (Agalychnis lemur) in Panama, was cultured and maintained on $1 \%$ tryptone agar plates. After $4 \mathrm{~d}$, zoospores were harvested by flooding the culture plate with sterile deionized water and incubating the plate at room temperature (RT) for $5 \mathrm{~min}$. After incubation, the liquid was removed using a sterile pipet and filtered through a sterile coffee filter to remove sporangia. The zoospore concentration was determined using a hemocytometer and averaging 3 replicate counts.

\section{Extraction methods}

Samples of 4 different zoospore quantities (referred to as 'zoospore levels' hereafter) were prepared from the zoospore solution. The total zoospore quantity in these 4 levels was 1000, 100, 10, and 1 zoospore. Each zoospore level was extracted 6 times with each tested extraction method, and 2 negative controls were extracted with each method. One of the negative controls included the deionized water used to harvest zoospores and the second included the nuclease-free water used to make the zoospore dilutions.

Three different extraction methods were tested: (1) PrepMan Ultra Reagent + Zirconium Beads, (2) Qiagen DNeasy Blood and Tissue Kit, and (3) Mobio PowerSoil DNA Isolation Kit. The PrepMan Ultra+ Zirconium Beads procedure originally described by Boyle et al. (2004) was performed in 2 ways: (1) using $50 \mu \mathrm{l}$ of PrepMan Ultra reagent and $30 \mathrm{mg}$ zirconium beads as used by Hyatt et al. (2007) (referred to as PrepMan-50 hereafter) and (2) using $100 \mu$ l of PrepMan Ultra reagent and $60 \mathrm{mg}$ zirconium beads (referred to as PrepMan-100 hereafter). The PrepMan-100 extraction was performed to make the extraction volume equivalent to that of the final elution step in the Mobio and Qiagen procedures. After extraction with PrepMan Ultra, a 1:10 dilution was made with an aliquot of the DNA extract, as this is commonly done to reduce PCR inhibition (e.g. Boyle et al. 2004, Woodhams et al. 2012a,b, Canestrelli et al. 2013, Rosa et al. 2013). The Mobio PowerSoil DNA 
Isolation was performed following the protocol used by Costello et al. (2009), with one modification: prior to the final elution, the spin column was incubated at RT for 5 min with the elution buffer (C6) to increase DNA yield. The Qiagen DNeasy procedure was performed according to the Animal Tissues protocol with the modifications outlined by Kosch \& Summers (2013). These include premixing the Buffer AL and $95 \% \mathrm{EtOH}$ prior to addition to the sample and incubating the spin column with Buffer AE for 5 min at RT prior to the final elution.

\section{qPCR analysis}

Extraction efficiency was evaluated using qPCR by amplifying the internal transcribed spacer (ITS)-5.8S rRNA region (Boyle et al. 2004) on a Biorad CFX96 Real-Time System machine. Each of the 6 extraction replicates from each extraction method was then run in triplicate qPCR reactions following the procedures of Boyle et al. (2004) with the following modification: the KlearKall Master Mix was used instead of the Taqman Universal Master Mix. For each qPCR reaction plate, a set of 6 standards (i.e. $10000,1000,100$, 10,1 , and 0.1 ) and a negative control were included in duplicate. For creating the standards, zoospores of $B d$ JEL423 were cultured, harvested, and counted as explained previously. A known quantity of zoospores $\left(1 \times 10^{6}\right)$ was extracted using the Qiagen DNeasy procedure explained above, and the dilutions of this extract were made to create each of the 6 standards.

\section{Inhibition testing}

Boyle et al. (2004) suggested that the PrepMan reagent itself can inhibit qPCR reactions. We tested this hypothesis by performing 2 additional qPCR reactions with exogenous internal positive controls (IPCs) as described by Hyatt et al. (2007) for 2 of the DNA extracts of each extraction method.

\section{Data analysis}

$B d$ was considered to be present in a sample if 2 of the $3 \mathrm{qPCR}$ replicates exhibited a fluorescence signal. Zoospore equivalents per sample were determined by averaging the triplicates and multiplying the direct qPCR zoospore estimates by 20 (DNeasy, Mobio, PrepMan-100d) or 10 (PrepMan-50d) to account for the whole extraction volume. For the Prep-
Man-50d and PrepMan-100d, zoospore equivalents were also multiplied again by 10 to account for the 1:10 dilution. Duplicate reactions run with IPCs were not included in the calculation of average zoospore equivalents per sample. Both presence/absence data and zoospore equivalent data were analyzed.

Statistical analyses were performed on the Qiagen DNeasy, Mobio Powersoil, and PrepMan-50d and 100d. Only the 1:10 dilutions were used because researchers typically implement this procedure during PCR testing to reduce inhibition from environmental factors. For all zoospore intensity analyses, zoospore equivalents +1 were $\log$ transformed to help achieve a normal distribution, which was successful for most treatments. To test whether there were differences between the extraction methods, 3 analyses were performed. First, to test for differences between the extraction methods across all zoospore levels as a whole, a 2-way ANOVA was performed on the log-transformed zoospore equivalents. Tukey post hoc tests were performed to determine which extraction methods differed. Second, in order to compare the extraction methods at each level independently, 1-way ANOVAs were performed on the logtransformed zoospore equivalents at each zoospore level, followed by Tukey post hoc tests. Third, to determine which methods were more efficient at detecting low levels of $B d$, presence-absence data at the level of 1 zoospore were evaluated using Fisher's exact tests. To assess inhibition associated with the Prepman reagent, we evaluated the quantification cycle (Cq) values of the VIC fluorescent marker, which corresponds to the signal from the internal positive control templates. The Cq values from the qPCR controls, PrepMan extracts, and the 1:10 dilutions of the PrepMan extracts were compared using a 1-way ANOVA. Cq values from each extraction method were pooled across zoospore levels, and for the controls, the Cq values of the standards and negative controls were pooled. All analyses were performed in R ver. 3.1.1 (R Core Team 2013).

\section{RESULTS}

The estimated zoospore equivalents differed among extraction methods $\left(F_{9,79}=23.116, \mathrm{p}<0.001\right)$. This difference was driven by the Qiagen DNeasy yielding significantly higher average zoospore equivalents that all other methods, and both PrepMan methods exhibiting higher average zoospore equivalents than Mobio PowerSoil (Tukey, p < 0.05). All 4 extraction methods showed a positive signal for $B d$ at 
the 1000 and 100 zoospore level (Table 1, Fig. 1); however, the estimated zoospore equivalents varied between extraction methods at these levels (1000 level, $F_{3,19}=5.64, \mathrm{p}=0.006 ; 100$ level, $F_{3,19}=8.35, \mathrm{p}=$ 0.0008). At the 1000 zoospore level, this difference was driven by Qiagen DNeasy and PrepMan-100d yielding significantly higher zoospore equivalents than Mobio PowerSoil, and at the 100 zoospore level, this difference was driven by Qiagen showing significantly higher zoospore equivalents than Mobio PowerSoil and PrepMan-50d (Tukey, p < 0.05). For the 10 zoospore level, both Qiagen DNeasy and Mobio PowerSoil extractions showed a positive signal for $B d$ in all 6 extraction replicates. However, for both PrepMan extractions (100 and $50 \mu \mathrm{l}), 5$ of the 6 replicates showed a positive signal for $B d$ (Table 1). The estimated zoospore equivalents differed significantly at the 10 zoospore level (ANOVA, $F_{3,19}=$ 15.575, p < 0.001), which was associated with Qiagen DNeasy estimating significantly greater zoospore equivalents than the other methods (Tukey, $\mathrm{p}<0.05$ ). For the single zoospore level, Qiagen DNeasy was effective at consistently yielding a positive $B d$ signal in $100 \%$ of the extraction replicates, Mobio PowerSoil showed a $B d$ positive signal in $50 \%$, and the PrepMan-50d and -100d extractions showed a $B d$ positive signal in $16.6 \%$ and $33.3 \%$, respectively (Table 1). The estimated zoospore equivalents differed significantly at the single zoospore level $\left(F_{3,19}=\right.$ 3.437, p = 0.03), which was associated with Qiagen DNeasy estimating significantly greater zoospore equivalents than Mobio PowerSoil (Tukey, p < 0.05). Importantly, there was significantly greater detection with Qiagen DNeasy in comparison to PrepMan$100 \mathrm{~d}$ at the single zoospore level (Fisher's exact test, $\mathrm{p}=0.01$ ). We acknowledge that in some cases the estimated zoospore equivalents exceeded the expected value based on the zoospore level. This result may be associated with differences in the ITS copy number in the standards and the samples.

Table 1. Detection ratio (DR = observed positives/expected positives) and estimated zoospore equivalents (ZEs) for each extraction method over the range of zoospore quantities. ZEs have been multiplied to account for total extraction volume. Standard error is provided in parentheses after each ZE

\begin{tabular}{|lcrrrrrrr|}
\hline $\begin{array}{l}\text { Extraction } \\
\text { method }\end{array}$ & \multicolumn{2}{c}{1000 zoospores } & \multicolumn{2}{c}{100 zoospores } & \multicolumn{2}{c}{10 zoospores } & \multicolumn{2}{c|}{1 zoospore } \\
& DR & ZE & DR & ZE & DR & ZE & DR & ZE \\
\hline Qiagen DNeasy & $6 / 6$ & $14726.05(7206.2)$ & $6 / 6$ & $267.08(32.02)$ & $6 / 6$ & $25.83(3.17)$ & $6 / 6$ & $4.47(1.39)$ \\
Mobio PowerSoil & $6 / 6$ & $1178.10(926.05)$ & $6 / 6$ & $39.28(26.4)$ & $6 / 6$ & $1.42(0.17)$ & $3 / 6$ & $0.12(0.06)$ \\
PrepMan-100 (1:10) & $6 / 6$ & $3989.69(1535.7)$ & $6 / 6$ & $63.99(10.05)$ & $5 / 6$ & $4.22(1.17)$ & $1 / 6$ & $0.53(0.36)$ \\
PrepMan-50 (1:10) & $6 / 6$ & $5612.55(2833.9)$ & $6 / 6$ & $50.6(14.40)$ & $5 / 6$ & $6.78(2.50)$ & $2 / 6$ & $0.23(0.23)$ \\
\hline
\end{tabular}

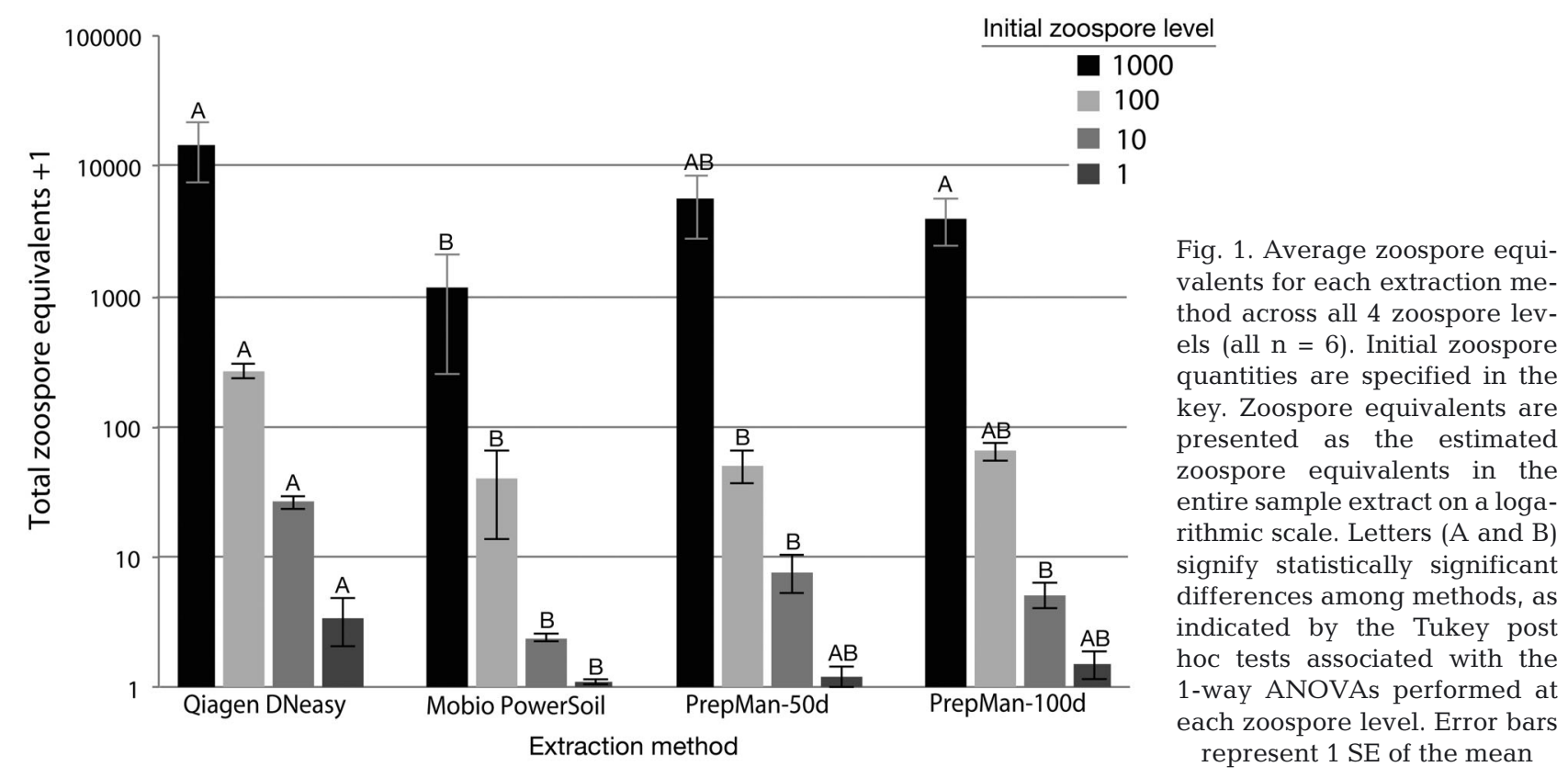




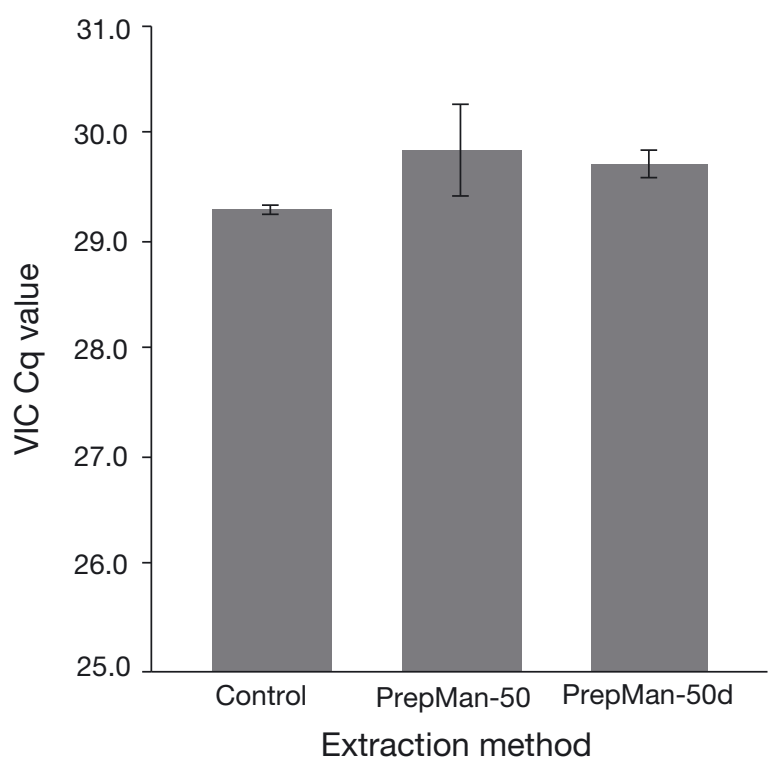

Fig. 2. Comparison of PCR inhibition among PrepMan methods. Average quantification cycle (Cq) values of the VIC fluorescence marker are given for the pooled control, PrepMan-50, and PrepMan-50 1:10 dilution reactions. Error bars represent $1 \mathrm{SE}$ of the mean

No PCR inhibition was detected in any extract, and all negative controls were negative. Importantly, there was no significant difference in the $\mathrm{Cq}$ values of the VIC fluorescent marker among the control samples, the PrepMan extracts, and their respective dilutions $\left(F_{2,50}=0.892, \mathrm{p}=0.416\right.$, Fig. 2$)$, which indicates that there is no inhibition in the samples extracted with this method.

\section{DISCUSSION}

Our results demonstrate that extraction efficiency varied among methods and that Qiagen DNeasy was the most efficient method for the detection of the amphibian pathogen $B d$ as it was able to detect all zoospore levels from 1000 to a single zoospore. In addition, it always exhibited the highest estimated zoospore equivalents, and in some cases estimated an order of magnitude more zoospore equivalents than the other methods, demonstrating it was able to recover the greatest amounts of the existing DNA. While Qiagen DNeasy clearly exhibited the highest efficiency in comparison to Mobio PowerSoil and PrepMan, the comparison of efficiency between Mobio PowerSoil and PrepMan depended on the metric of analysis. In terms of zoospore intensity, Mobio PowerSoil generally yielded lower zoospore equivalents in comparison to PrepMan. However, in terms of detecting the presence of $B d$, Mobio PowerSoil performed better than PrepMan. At the single zoospore level, Mobio PowerSoil failed to detect Bd 50\% of the time whereas the PrepMan-50d and PrepMan$100 d$ extractions failed to detect $B d 83.3 \%$ and $66.7 \%$ of the time, respectively. In addition, at the 10 zoospore level, Mobio detected Bd all the time while both PrepMan extractions were unable to detect $B d 17 \%$ of the time. The observed differences in extraction efficiency may be associated with the different mechanisms of extraction of these methods. Qiagen uses only chemical lysis, while Mobio Powersoil and the PrepMan + Zirconium methods use mechanical and chemical lysis steps.

Our results parallel those observed in other studies. In a recent study related to DNA extraction from human fecal samples, PowerSoil exhibited one of the lowest amounts of DNA recovered (Claassen et al. 2013). Mobio PowerSoil is specially designed to remove inhibitors in the environment, such as humic acid, and has 3 precipitation and 5 spin column steps to clean and purify the DNA. These steps can also lead to DNA loss, which could explain the lower DNA recovery observed. Similarly, in a study evaluating pathogenic bacterial detection, PrepMan extraction exhibited one of the lowest DNA recoveries (Brewster \& Paoli 2013).

High extraction efficiency is crucial for accurately diagnosing the presence of $B d$ as well as other pathogens that use similar methods for detection. This is especially true when prevalence and infection intensity are expected to be low. For example, when monitoring Bd-naïve areas, such as Papua New Guinea and regions of Madagascar, for the arrival of the pathogen (Dahl et al. 2012, Vredenburg et al. 2012, Weldon et al. 2013), it is imperative to be able to detect low-intensity infections in order to detect $B d$ at its earliest arrival so proactive actions in response to the presence of $B d$ can be implemented promptly. High efficiency is also important in other situations where pathogen prevalence and intensity are expected to be low, such as enzootic situations where $B d$ may be out of its temperature optimum or with resistant species (Rothermel et al. 2008, Rebollar et al. 2014). Additionally, in order to accurately monitor disease dynamics, such as seasonal fluctuations, it is important to accurately measure prevalence and infection intensity. Swabbing has been shown to underestimate infection intensity compared to a bath water filtration method (Shin et al. 2014). Thus, if swabbing is used, it becomes important not to further underestimate infection intensity by using a less sensitive extraction protocol. PrepMan Ultra + Zirco- 
nium, recommended by Boyle et al. (2004) and the method of choice for many researchers, was one of the least efficient of the tested methods. Therefore, in previous $B d$ surveys that used PrepMan extraction methods, prevalence and infection intensity may have been underestimated, which could have consequences for accurately understanding $B d$ dynamics or detecting $B d$ earlier in its emergence.

All extraction methods yielded a positive $B d$ signal when the zoospore amount was fairly high (i.e. at the 100 and 1000 zoospore quantities). Therefore, in situations where infection intensity is expected to be high, any extraction method could be used for detecting $B d$ presence. However, our results show that different extraction efficiencies of these methods can lead to different estimated zoospore equivalents from the same original zoospore concentration. For example, estimated zoospore equivalents based on Qiagen DNeasy extractions were at times an order of magnitude higher compared to Mobio PowerSoil extractions. In light of these findings, it is important to take into consideration the extraction method when comparing across studies, and when extraction methods differ, conclusions should be drawn with caution. Other factors, such as the $B d$ strain used as the reference strain (Longo et al. 2013) and storage methods (Hyatt et al. 2007), can also affect infection intensity estimates, and therefore need to be considered when comparing across studies. Additionally, to reduce overor underestimation of zoospore values, it will be important to use the same extraction method for the standards and the samples obtained from the field. To understand natural fluctuations in infection intensities in field and experimental settings, it appears that Qiagen DNeasy will provide the most accurate infection intensity values due to its superior extraction efficiency.

Importantly, for some extraction methods at the 10 and single zoospore concentration, the calculated zoospore equivalents estimated from the qPCR reaction were $<1$ (Table 1). This result demonstrates the importance of considering values $<1$ to be true positives. Many previous studies have disregarded such values as true positives (e.g. Kriger \& Hero 2007, Kriger et al. 2007, Kilburn et al. 2010, Woodhams et al. 2012a, 2014). Considering these values as true positives will reduce the risk of false negatives. It will be important when considering signals $<1$ to look also at consistency of the signal in order to minimize false positives. In the collected data, all observed low signals were consistent (i.e. 2 of the 3 replicates showed a fluorescence signal). If a weak signal occurs in only 1 replicate of the $\mathrm{qPCR}$ reaction, then it may be a false positive. To balance the risks of false negatives and false positives, consistent, low signals (i.e. estimates $<1)$ should be considered a true positive and will enable the most accurate diagnosis of infection status.

Boyle et al. (2004) suggested that high concentrations of PrepMan Ultra reagent are inhibitory in downstream GPCR reactions; however, our results showed no inhibition in both the 'full strength' or diluted PrepMan extracts. Perhaps this was due to the use of a different Taq enzyme in the master mix for the qPCR reaction. In light of this finding, it may be possible to use undiluted PrepMan extracts, which would maintain higher levels of DNA for downstream reactions. PrepMan Ultra employs no DNA clean-up steps, so it is possible that inhibition of qPCR could still result from environmental contaminants in field-collected samples using this technique. If there is inhibition as a result of the field sample, methods such as the use of BSA could be employed to reduce inhibition for qPCR (Kosch \& Summers 2013). Such a method would eliminate the need to dilute the DNA extract.

It is important to consider the cost of sampling processing when deciding on extraction method. PrepMan, Qiagen DNeasy, and Mobio PowerSoil cost US $\$ 0.26, \$ 2.66$, and $\$ 4.94$ per sample, respectively, at the time of this study. While PrepMan has the lowest cost, it lacks extraction efficiency for low levels of DNA. Mobio PowerSoil is the most expensive and has lower extraction efficiency. Qiagen DNeasy is intermediate in cost and has the greatest efficiency. Similarly, Qiagen DNeasy was recommended as the ideal method for removing PCR inhibitors, and therefore has the optimal balance of removing inhibitors (Kosch \& Summers 2013), high extraction efficiency, and cost effectiveness.

\section{CONCLUSIONS}

We conclude that Qiagen DNeasy is the most efficient of the 3 extraction methods tested since low levels of $B d$ could be detected reliably only with this method. This is important when $B d$ is emerging in naïve areas or in other situations when its presence is expected to be rare. These findings demonstrate the importance of having high extraction efficiency in order to diagnose disease early and be able to effectively respond to any emerging disease threat. This principle extends beyond the detection of the amphibian chytrid fungus and will be important to consider when monitoring other wildlife and human pathogens. 
Acknowledgements. We thank Joyce Longcore for providing us with the $B d$ strain used for this study. This investigation was funded by NSF grant 1136602 awarded to R.N.H.

\section{LITERATURE CITED}

Annis SL, Dastoor FP, Ziel H, Daszak P, Longcore JE (2004) A DNA-based assay identifies Batrachochytrium dendrobatidis in amphibians. J Wildl Dis 40:420-428

Boyle DG, Boyle DB, Olsen V, Morgan JAT, Hyatt AD (2004) Rapid quantitative detection of chytridiomycosis (Batrachochytrium dendrobatidis) in amphibian samples using real-time Taqman PCR assay. Dis Aquat Org 60:141-148

> Brewster JD, Paoli GC (2013) DNA extraction protocol for rapid PCR detection of pathogenic bacteria. Anal Biochem 442:107-109

> Canestrelli D, Zampiglia M, Nascetti G (2013) Widespread occurrence of Batrachochytrium dendrobatidis in contemporary and historical samples of the endangered Bombina pachypus along the Italian peninsula. PLoS ONE 8:e63349

> Chatfield MWH, Brannelly LA, Robak MJ, Freeborn L, Lailvaux SP, Richards-Zawacki CL (2013) Fitness consequences of infection by Batrachochytrium dendrobatidis in northern leopard frogs (Lithobates pipiens). EcoHealth 10:90-98

> Cheng TL, Rovito SM, Wake DB, Vredenburg VT (2011) Coincident mass extirpation of neotropical amphibians with the emergence of the infectious fungal pathogen Batrachochytrium dendrobatidis. Proc Natl Acad Sci USA 108:9502-9507

> Claassen S, du Toit E, Kaba M, Moodley C, Zar HJ, Nicol MP (2013) A comparison of the efficiency of five different commercial DNA extraction kits for extraction of DNA from faecal samples. J Microbiol Methods 94:103-110

Costello EK, Lauber CL, Hamady M, Fierer N, Gordon JI, Knight R (2009) Bacterial community variation in human body habitats across space and time. Science 326: 1694-1697

> Crawford AJ, Lips KR, Bermingham E (2010) Epidemic disease decimates amphibian abundance, species diversity, and evolutionary history in the highlands of central Panama. Proc Natl Acad Sci USA 107:13777-13782

Dahl C, Kiatik I, Bronikowski E, Robert C and others (2012) Batrachochytrium dendrobatidis not found in rainforest frogs along an altitudinal gradient of Papua New Guinea. Herpetol J 22:183-186

Duncan Pullen K, Best AM, Ware JL (2010) Amphibian pathogen Batrachochytrium dendrobatidis prevalence is correlated with season and not urbanization in central Virginia. Dis Aquat Org 91:9-16

> Fisher MC, Garner TWJ, Walker SF (2009) Global emergence of Batrachochytrium dendrobatidis and amphibian chytridiomycosis in space, time, and host. Annu Rev Microbiol 63:291-310

Fisher MC, Henk DA, Briggs CJ, Brownstein JS, Madoff LC, McCraw SL, Gurr SJ (2012) Emerging fungal threats to animal, plant and ecosystem health. Nature 484:186-194

> Harris RN, Brucker RM, Walke JB, Becker MH and others (2009) Skin microbes on frogs prevent morbidity and mortality caused by a lethal skin fungus. ISME $\mathrm{J} 3$ : 818-824

> Hoffmann M, Hilton-Taylor C, Angulo A, Böhm M and others (2010) The impact of conservation on the status of the world's vertebrates. Science 330:1503-1509

Hyatt AD, Boyle DG, Olsen V, Boyle DB and others (2007) Diagnostic assays and sampling protocols for the detection of Batrachochytrium dendrobatidis. Dis Aquat Org 73:175-92

Kilburn VL, Ibáñez R, Sanjur O, Bermingham E, Suraci JP, Green DM (2010) Ubiquity of the pathogenic chytrid fungus, Batrachochytrium dendrobatidis, in anuran communities in Panamá. EcoHealth 7:537-548

> Kirshtein JD, Anderson CW, Wood JS, Longcore JE, Voytek MA (2007) Quantitative PCR detection of Batrachochytrium dendrobatidis DNA from sediments and water. Dis Aquat Org 77:11-15

Kosch TA, Summers K (2013) Techniques for minimizing the effects of PCR inhibitors in the chytridiomycosis assay. Mol Ecol Resour 13:230-236

- Kriger KM, Hero JM (2007) The chytrid fungus Batrachochytrium dendrobatidis is non-randomly distributed across amphibian breeding habitats. Divers Distrib 13:781-788

Kriger KM, Pereoglou F, Hero JM (2007) Latitudinal variation in the prevalence and intensity of chytrid (Batrachochytrium dendrobatidis) infection in eastern Australia. Conserv Biol 21:1280-1290

Longo AV, Rodriguez D, da Silva Leite D, Toledo LF, Mendoza Almeralla C, Burrowes PA, Zamudio KR (2013) ITS1 copy number varies among Batrachochytrium dendrobatidis strains: implications for qPCR estimates of infection intensity from field-collected amphibian skin swabs. PLoS ONE 8:e59499

McKenzie VJ, Bowers RM, Fierer N, Knight R, Lauber CL (2012) Co-habiting amphibian species harbor unique skin bacterial communities in wild populations. ISME J 6: 588-596

Muletz CR, Myers JM, Domangue RJ, Herrick JB, Harris RN (2012) Soil bioaugmentation with amphibian cutaneous bacteria protects amphibian hosts from infection by $\mathrm{Ba}$ trachochytrium dendrobatidis Biol Conserv 152:119-126

R Core Team (2013) R: a language and environment for statistical computing. R Foundation for Statistical Computing, Vienna. www.r-project.org

Rasmussen C, Eisenberg T, Alfermann D, Köhler J (2012) Presence of Batrachochytrium dendrobatidis in amphibians from central and southern Hesse, central Germany: results from a preliminary regional screening. Salamandra 48:166-172

> Rebollar EA, Hughey MC, Harris RN, Domangue RJ, Medina D, Ibáñez R, Belden LK (2014) The lethal fungus Batrachochytrium dendrobatidis is present in lowland tropical forests of far eastern Panamá. PLoS ONE 9: e95484

Rosa GM, Anza I, Moreira PL, Conde J, Martins F, Fisher MC, Bosch J (2013) Evidence of chytrid-mediated population declines in common midwife toad in Serra da Estrela, Portugal. Anim Conserv 16:306-315

> Rothermel BB, Walls SC, Mitchell JC, Dodd CK Jr and others (2008) Widespread occurrence of the amphibian chytrid fungus Batrachochytrium dendrobatidis in the southeastern USA. Dis Aquat Org 82:3-18

Searle CL, Gervasi SS, Hua J, Hammond JI, Relyea RA, Olson DH, Blaustein AR (2011) Differential host susceptibility to Batrachochytrium dendrobatidis, an emerging amphibian pathogen. Conserv Biol 25:965-974

> Shin J, Bataille A, Kosch TA, Waldman B (2014) Swabbing often fails to detect amphibian chytridiomycosis under conditions of low infection load. PLoS ONE 9:e111091 
Swei A, Rowley JJL, Rödder D, Diesmos MLL and others (2011) Is chytridiomycosis an emerging infectious disease in Asia? PLoS ONE 6:e23179

Vredenburg VT, Preez L, Raharivololoniaina L, Vieites DR, Vences M, Weldon C (2012) A molecular survey across Madagascar does not yield positive records of the amphibian chytrid fungus Batrachochytrium dendrobatidis. Herpetol Notes 5:507-517

Wake DB, Vredenburg VT (2008) Are we in the midst of the sixth mass extinction? A view from the world of amphibians. Proc Natl Acad Sci USA 105:11466-11473

Weldon C, Crottini A, Bollen A, Rabemananjara FCE, Copsey J, Garcia G, Andreone F (2013) Pre-emptive national monitoring plan for detecting the amphibian chytrid fun-

Editorial responsibility: Louise Rollins-Smith, Nashville, Tennessee, USA gus in Madagascar. EcoHealth 10:234-240

> Woodhams DC, Bell SC, Kenyon N, Alford RA, Rollins-Smith LA (2012a) Immune evasion or avoidance: fungal skin infection linked to reduced defence peptides in Australian green-eyed treefrogs, Litoria serrata. Fungal Biol 116:1203-1211

Woodhams DC, Bigler L, Marschang R (2012b) Tolerance of fungal infection in European water frogs exposed to Batrachochytrium dendrobatidis after experimental reduction of innate immune defenses. BMC Vet Res 8:197

Woodhams DC, Brandt H, Baumgartner S, Kielgast J and others (2014) Interacting symbionts and immunity in the amphibian skin mucosome predict disease risk and probiotic effectiveness. PLoS ONE 9:e96375

Submitted: May 12, 2014; Accepted: November 4, 2014 Proofs received from author(s): January 30, 2015 